\title{
Development of a Fast-Spectrum Self-Powered Neutron Detector for Molten Salt Experiments in the Versatile Test Reactor
}

\author{
K. C. Goetz ${ }^{1 *}$, S. M. Cetiner ${ }^{2}$, C. Celik ${ }^{1}$ and J. $\mathrm{Hu}^{1}$ \\ ${ }^{1}$ Oak Ridge National Lab, USA \\ ${ }^{2}$ Idaho National Lab, Massachussets Institute of Technology, USA
}

Corresponding author: goetzkc@ornl.gov

\begin{abstract}
The self-powered neutron detector (SPND) is a widely used flux monitor in thermal nuclear reactors. Although this is a mature technology, the current state of the art is tuned for a thermal neutron spectrum, so many of the devices currently in use lack sensitivity to fast neutrons. Because current in SPNDs is produced through nuclear reactions with the neutron flux inside a reactor, sensitivity in SPNDs is determined by the neutron cross section of the neutron-sensitive portion of the detector, termed the emitter. This neutron cross section drops by orders of magnitude between thermal and fast neutron energies for many emitters in currently used SPNDs, with a corresponding drop in current from the detector. This paper discusses efforts to develop a fast-spectrum self-powered neutron detector (FS-SPND) that is sensitive to neutrons with energies ranging from $0.025 \mathrm{eV}$ up to $1 \mathrm{MeV}$. An in-depth analysis of Evaluated Nuclear Data File (ENDF)/B-VII.1 neutron-capture cross sections was performed, and four new materials were identified that are suitable emitter candidates for use in measuring fast neutrons. All four materials are stable mid-shell nuclei in the region between doubly magic ${ }^{132} \mathbf{S n}$ and ${ }^{208} \mathbf{P b}$. Each candidate was simulated with the Geant4 Monte Carlo simulation toolkit to optimize overall detector efficiency.
\end{abstract}

Index Terms-fast-spectrum nuclear reactor, instrumentation and controls, nuclear reactor, self-powered neutron detector, sodium-cooled fast reactor, Versatile Test Reactor

\section{INTRODUCTION}

Self-powered neutron detectors (SPNDs) have been a common diagnostic tool for intra-core neutron flux mapping in thermal nuclear reactors for more than 60 years [1], [2]. They are useful flux monitors because they are durable, compact, and simple, and they produce a signal proportional to local neutron flux without requiring an external source of power. Signal in these detectors is a current source driven by the electrons generated from nuclear reactions within the emitter that make it out to the collector. A diagram of a generic SPND is shown in Figure 1.

Some common SPND emitters are vanadium, rhodium, silver, and cobalt. SPND insulators are generally made of magnesium oxide, aluminum oxide, or are a vacuum. SPND

This manuscript has been authored by UT-Battelle, LLC, under contract DE-AC05-00OR22725 with the US Department of Energy (DOE). The US government retains and the publisher, by accepting the article for publication, acknowledges that the US government retains a nonexclusive, paid-up, irrevocable, worldwide license to publish or reproduce the published form of this manuscript, or allow others to do so, for US government purposes. DOE will provide public access to these results of federally sponsored research in accordance with the DOE Public Access Plan (http://energy.gov/downloads/doepublic-access-plan).

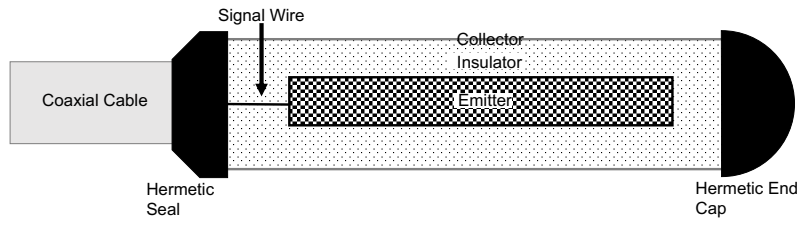

Fig. 1. Diagram of an SPND. It is expected that neutrons will be captured or scattered within the emitter (checked region), resulting in nuclear or ionization reactions that produce electrons with energies high enough that they can escape the emitter and travel through the insulator (white dotted region) to the collector (gray region).

collectors and signal wires are most often made of Inconel steel.

\section{Self-Powered Neutron Detector Types}

The two types of SPNDs-delayed and prompt-are distinguished by the reaction mechanism producing the majority of the electrical signal in the detector. A single SPND will have a delayed component to its signal, as well as a prompt component. Delayed-type SPNDs produce a signal through a $(n, \beta)$ reaction. In this reaction, the incoming neutron is captured, and the subsequent reaction product $\beta$ decays, emitting a $\beta$ particle (electron). The electron produces signal in the detector when it exits the emitter and travels to the collector. SPNDs are generally read out by a current meter at the end of a long coaxial cable well outside reactor containment. Delayedtype SPNDs are so named because the response time of the detector to changed in neutron flux depends on the $\beta$-decay half life of the neutron capture reaction product.

Alternately, prompt-type SPNDs generate an electrical current through a $(n, \gamma)\left(\gamma, e^{-}\right)$reaction series. In this reaction series, a neutron is captured or scattered, and the resulting nucleus de-excites through $\gamma$-ray emission. Current is produced when the resultant $\gamma$ ray knocks an electron out of the neighboring atom, and that electron is absorbed in the collector. Prompt-type SPNDs respond instantaneously to changes in neutron flux.

\section{FASt-Spectrum SElF-Powered NeUtron DETECTORS}

Next-generation reactors are on the horizon. Because some next-generations reactors are designed to operate with harder neutron spectra peaking around $0.5 \mathrm{MeV}$, diagnostic tools that 
are tuned to their neutron spectra must be developed. Faster neutron spectra are of salient concern for detectors such as SPNDs, in which the signal is driven by nuclear reactions, and for which the state-of-the-art is tuned for sensitivity to a thermal neutron spectrum. This is because the neutron cross section for many materials can drop by orders of magnitude between thermal and fast neutron energies.

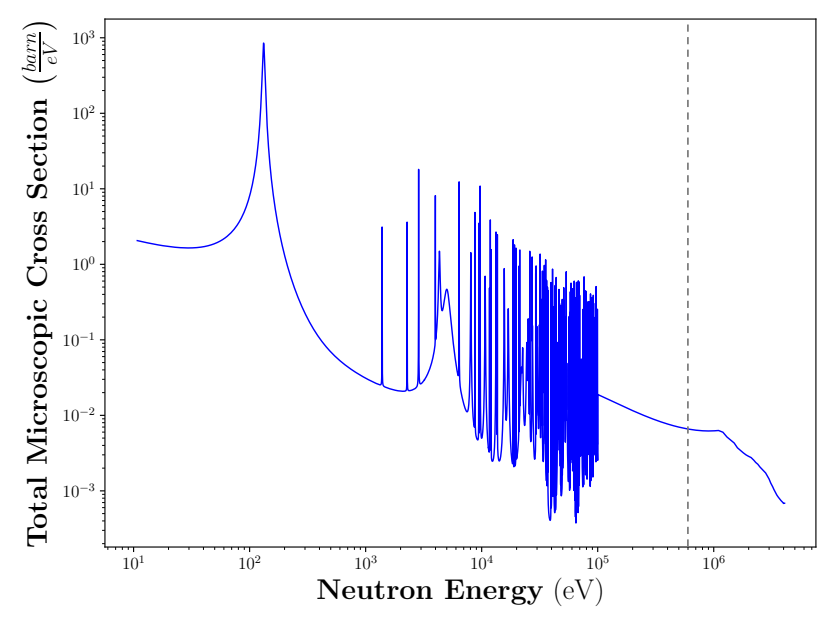

Fig. 2. The total microscopic $(n, \gamma)$ cross section for ${ }^{59} \mathrm{Co}$ from the ENDF/BVII.1 library, courtesy of the NNDC [3], [4]. The gray dotted line represents the peak neutron energy in an SFR spectrum at $0.5 \mathrm{MeV}$. Peaks in the neutron cross section, also termed resonances, correspond to nuclear levels. ${ }^{59} \mathrm{Co}$ displays the typical structure wherein at low energies the distance between levels is large (resonance at $\sim 100 \mathrm{eV}$ ) followed by an increase in level density $(1-100 \mathrm{keV})$ resulting in an eventual continuum $(i, 100 \mathrm{keV})$ where individual levels can no longer be resolved.

One example of this is shown in Figure 2 for ${ }^{59} \mathrm{Co}$, a commonly used emitter material in SPNDs for thermal nuclear reactors. The neutron cross section for ${ }^{59} \mathrm{Co}$ drops by multiple orders of magnitude between thermal energies and $0.5 \mathrm{MeV}$ with a corresponding decrease in detector sensitivity.

\section{AnAlysis and Material SElection}

\section{A. Analysis of ENDF Cross sections}

The object of this work is to identify new and currently used emitter materials that will have the requisite sensitivity for operation within a fast-spectrum nuclear reactor. Requisite sensitivity is defined here as having an expected output current while operating in a fast reactor flux that is comparable to that of a state-of-the-art thermal-spectrum SPND operating in a thermal reactor flux. To that end, as part of the emitter selection process a generic neutron spectrum from a sodiumcooled fast reactor was used [5]. This flux spectrum was used in conjunction with $(n, \gamma)$ cross section data from the ENDF library for the full range of stable nuclei to calculate weighted total microscopic $(n, \gamma)$ cross sections as shown in Eq. (1) [6], [7]:

$$
\sigma_{\xi}=\frac{\int_{E_{S F R}} d E \phi(E) \sigma_{\xi}(E)}{\int_{E_{S F R}} d E \phi(E)},
$$

where $\sigma_{\xi}$ is the microscopic cross section for reaction type $\xi$ integrated over the full energy range of the sodium-cooled fast reactor (SFR) neutron flux, $\mathrm{E}_{S F R} . \sigma_{\xi}(E)$ is the energydependent microscopic cross section for reaction type $\xi$, in this case total $(n, \gamma)$, from the ENDF/B-VII.1, ENDF/B-VIII.0, or JEFF-3.3 libraries [3]. $\phi(E)$ is the scalar neutron flux [5]. An example of the result of the process is shown in Figure 3 for ${ }^{51} \mathrm{~V}$, another common SPND emitter.

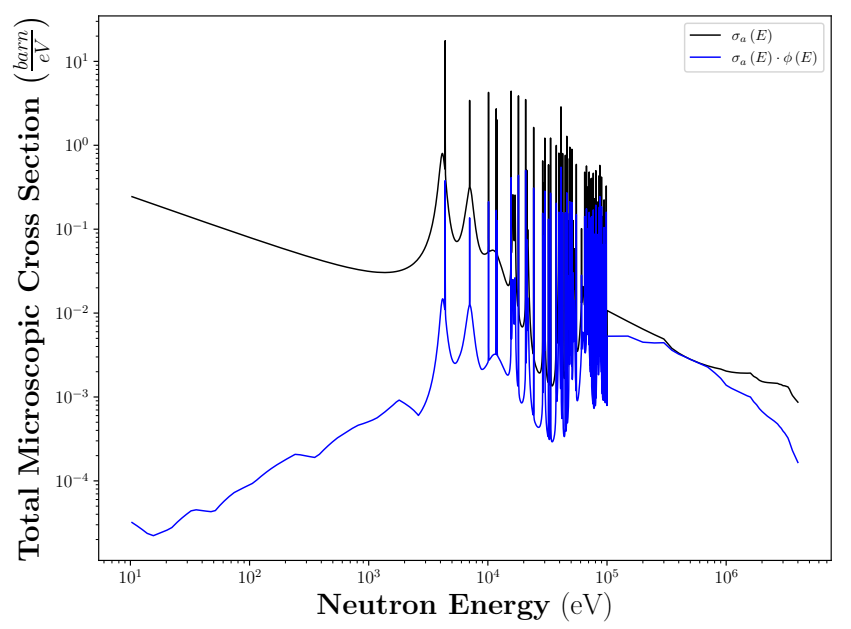

Fig. 3. Microscopic cross section data for ${ }^{51}$ Va from ENDF/B-VII.1 (black) and the resultant calculated cross section data (blue) taking into account the SFR neutron flux spectrum [3], [5].

The macroscopic cross section $\Sigma_{\xi}$ representing all $(n, \gamma)$ interactions per unit path length traveled in a given medium and integrated over the entire SFR spectrum was then calculated via the following expression:

$$
\Sigma_{\xi}=N \sigma_{\xi}
$$

where $N$ is atomic number density of the element. For elements with more than one stable isotope, $\Sigma_{\xi}$ was calculated for each individually.

Reaction and burnup rates are gauges for the sensitivity of an eventual FS-SPND. To that end, the time-dependent reaction rate $R_{\xi}(t)$ for a given reaction-type $\xi$ was calculated with

$$
R_{\xi}(t)=\int_{E_{S F R}} d E N(t) \phi(E, t) \sigma_{\xi}(E)
$$

where $N(t)$ is the time-dependent atomic number density for the material, and $\phi(E, t)$ is the time-dependent scalar flux.

The time-dependent burnup rate, $b(t)$, of the emitter material at a given time $t$ is defined as

$$
b(t)=\int_{E_{S F R}} d E N(t) \phi(E, t) \sigma_{a}(E),
$$

where $\sigma_{a}(E)$ is the neutron absorption cross section for a given stable isotope. Similarly, the time-integrated burnup, 
$B(t)$, at a given time $t$ is given by

$$
B(t)=\int_{0}^{t} d \tau b(\tau)=\int_{0}^{t} d \tau N(\tau) \int_{E_{S F R}} d E \phi(E, \tau) \sigma_{a}(E) .
$$

The analysis was verified through a comparison of the calculated burnup rate for ${ }^{103} \mathrm{Rh}$ in a thermal neutron flux to that in literature. A burnup rate of $0.39 \%$ was calculated, which matches the published value [8].

This stage of the analysis yielded a list of elements with a sensitivity to the neutron flux in an SFR on par with rhodium, a highly sensitive emitter, in a thermal nuclear reactor. Criterion included total macroscopic cross section (Equation 2) and burn up rate (Equation 4). The list is as follows: rhenium, iridium, osmium, europium, lutetium, tantalum, gadolinium, terbium, tungsten, platinum, palladium, silver, rhodium, technetium, thallium, thulium, ytterbium, erbium, dysprosium, and hafnium.

\section{B. General Selection Criteria}

However, sensitivity to fast neutrons is not the only concern when choosing an appropriate emitter material for use in nextgeneration nuclear reactors. The materials must have a high enough melting point to survive within an operating nuclear reactor core. This requirement led to selection of materials with melting points above $700^{\circ} \mathrm{C}$ [9]. Because a simpler detector response to changes in neutron flux is preferred, materials with one or two single isotopes were selected. The final general criterion was that all materials must be both readily available and machinable to facilitate fabrication of the end detector. These constraints pared down the list to rhenium, iridium, lutetium, tantalum, terbium, silver, rhodium, and thulium.

\section{Physics-based Selection Criteria}

Following down-selection according to the general criteria listed above, further constraints must be applied to minimize background noise and maximize efficiency in the detector.

For prompt-type SPNDs, the $\beta$-decay half life of the neutron-capture reaction product should be maximized, on the order of months. With an ideal prompt-type emitter having a delayed response time that is on the same order of magnitude as the expected reactor cycle. This timeframe was chosen to minimize the introduction of an ever-growing delayed component to the detector response as reaction products continue to build up and decay within the emitter. In an improperly chosen emitter material, this delayed component would act as undesirable background noise that could eventually overwhelm the preferred $\left(\gamma, e^{-}\right)$reaction channel. Additionally, to maximize detector efficiency, prompt SPNDs should have a large atomic number $(Z)$. This serves to have an appropriate number of electrons available for the signal-producing reaction in the detector.

In delayed-type SPNDs it is desirable to minimize the response time of the detector to changes in neutron flux. Because signal in the detector is produced through an $(n, \beta)$ reaction, this is achieved through selecting materials for which the neutron-capture reaction product has as a $\beta$-decay half life that is as short as possible, preferably on the order of seconds or less. For this SPND type, detector efficiency is maximized by selecting emitter materials that will produce higher energy $\beta$ particles, thus increasing the likelihood that the electron will have enough energy to escape the emitter and travel out to the collector. This is achieved by selecting materials for which the neutron-capture reaction product has a high $Q_{\beta}$ value and strong feeding to the ground or low excited states in the $\beta$ decay daughter.

Rhenium was rejected at this stage because the $\beta$-decay half lives of the neutron-capture reaction products were unsuitable for either a prompt or delayed SPND. The two stable rhenium isotopes, ${ }^{185} \mathrm{Re}$ and ${ }^{187} \mathrm{Re}$, neutron capture to ${ }^{186} \mathrm{Re}$ and ${ }^{188} \mathrm{Re}$, respectively. ${ }^{186} \operatorname{Re}$ has a $\beta$-decay half life of 3.7 days, whereas the $\beta$-decay half life of ${ }^{188} \operatorname{Re}$ is 17 hours. This disqualifies rhenium as a candidate, because these response times are far too short to be a reasonable prompt-type SPND and far too long for a delayed-type SPND.

It should be noted however, that many of the rejected candidates for this work are currently used in SPNDs, i.e. hafnium and platinum. Therefore the disqualifications of materials discussed here should be viewed more as guidelines with materials rejected based on the understanding that they are less ideal SPND emitters than the elected candidates.

\section{Geant4 Model of SPNDs}

A detector inside the core of an operating nuclear reactor will experience more than just the neutron flux accounted for in the analysis up to this point. The $\gamma$ flux inside the core will induce a prompt signal in both prompt- and delayedtype SPNDs through a $\left(\gamma, e^{-}\right)$reaction. Depending on the application and detector type, this signal may or may not be a desirable component of the overall detector response. Additionally, the high-temperature environment inside the core will cause changes to the neutron cross section through Doppler broadening [10]. To maximize efficiency for each SPND emitter, the geometry of each SPND must be tuned to account for the difference in its respective electron spectrum. Therefore, to appropriately optimize any future FS-SPNDs ahead of a costly, time-consuming fabrication process, it is beneficial to first simulate each in a Monte Carlo code such as Geant4.

To that end, a model of an generic SPND was developed using Geant4 version 10.6.1 [8]. A visualization of the model is shown in Figure 4.

This model takes prompt $\gamma$ and neutron spectra as an input to the simulation, as well as irradiation "time." This "time" allows for the grow-in and decay-of-neutron-capture reaction products to reproduce the effects of burn-up within the detector. An additional feature in the simulation is the use of the neutron cross section database generated for Geant4 using ENDF/B-V11.1 by Mendoza et al. [3], [11]. The data are further broadened using AMPX-6, allowing an irradiation temperature to be established within the simulation space to more accurately reproduce material changes inside an operating SFR [10], [12]. The model was verified by simulating a 


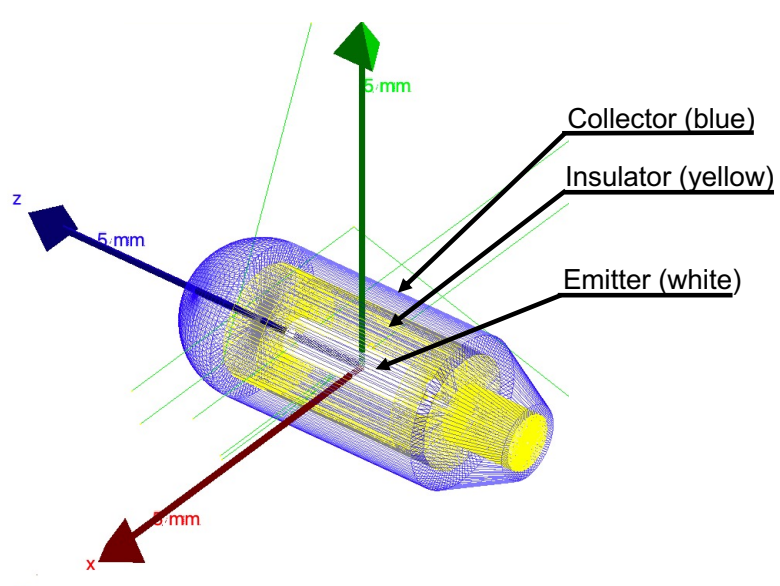

Fig. 4. Visualization of an SPND from within the simulation shown as a wire frame to allow a view inside the detector. The white at the center of the detector is the emitter, the yellow is insulator, and the blue is the collector. The green lines are reactor $\gamma$ rays impinging the detector.

rhodium SPND in a field of thermal neutrons. The calculated response time of the detector was 4.8 minutes, falling within the expected 3-5 minute window [2].

\section{E. Geant4 Analysis}

After the candidate list was narrowed down to iridium, lutetium, tantalum, terbium, silver, rhodium, and thulium, each material was simulated using the Geant4 SPND model, assuming natural elemental abundances. The insulator and collectors were the same materials for all runs, $\mathrm{MgO}$ and Inconel 600, respectively. The environment immediately around the detector was simulated as a pipe filled with magnesium salt. An SFR environment was assumed, with prompt neutrons and $\gamma$ fluxes from Verma et al., and an irradiation temperature of $400{ }^{\circ} \mathrm{C}$ [5]. All incoming particles were shaped into a rectangular beam and aimed at the emitter to reduce the number of events needed in the simulation. The irradiation time was set to 0 seconds to act as a reactor impulse at time $t=0$. Reaction products were allowed to grow in and decay away until $t=\infty$. All reaction channels were allowed in the simulation, including any that might compete with the desired signal. At this stage, no attempt was made to monitor electron movement between the emitter to the collector, as that optimization is beyond the scope of this work. The results from these efforts are a tally of the number of electrons generated in the emitter for each SPND to provide a direct comparison of emitter materials.

\section{Results}

Five emitter candidates were identified for prompt-type SPNDs, four of which are new, and two of which are suitable for delayed-type SPNDs, and both of which are well known SPND emitters. The prompt-type emitter materials are shown in Table I, and the delayed-type materials are shown in Table II. The results are listed in order of preference. Suitability is judged based on the total macroscopic cross section, the number and relative abundance of stable isotopes, the $\beta$ decay half lives of the reaction products, as well as calculated reaction and burnup rates.
TABLE I

TOP EMITTER CANDIDATES FOR PROMPT-TYPE SPNDS LISTED IN ORDER OF SUITABILITY. ALL CANDIDATES ARE NEWLY IDENTIFIED MATERIALS. $\mathrm{T}_{\frac{1}{2}}$ REPRESENTS THE $\beta$-DECAY HALF LIFE OF THE REACTION PRODUCT, BURNUP $(\Delta)$ IS CALCULATED ASSUMING $\phi=5 \times 10^{15} \mathrm{~N} / \mathrm{S}^{2} \mathrm{CM}^{2}$.

\begin{tabular}{llll}
\hline Nucleus & $\Sigma\left(\frac{1}{\mathrm{~cm}^{2}}\right)$ & $\begin{array}{l}\Delta / \text { month at } \\
0.5 \mathrm{MeV}(\%)\end{array}$ & $\mathrm{t}_{\frac{1}{2}}$ \\
\hline${ }^{181} \mathrm{Ta}$ & 13372 & 0.21 & 114 days \\
${ }^{159} \mathrm{~Tb}$ & 13423 & 0.40 & 72 days \\
${ }^{169} \mathrm{Tm}$ & 8043 & 0.25 & 128 days \\
${ }^{176} \mathrm{Lu}$ & 16500 & 0.75 & 6.6 days \\
${ }^{191} \mathrm{Ir}$ & 25281 & 0.34 & 73 days \\
\hline
\end{tabular}

TABLE II

TOP EMITTER CANDIDATES FOR DELAYED-TYPE SPNDS LISTED IN ORDER OF SUITABILITY. T $\frac{1}{2}$ REPRESENTS THE $\beta$-DECAY HALF LIFE OF THE REACTION PRODUCT, BURNUP $(\Delta)$ IS CALCULATED ASSUMING $\phi=5 \times 10^{15} \mathrm{~N} / \mathrm{s} / \mathrm{CM}^{2}$

\begin{tabular}{llll}
\hline Nucleus & $\Sigma\left(\frac{1}{c m^{2}}\right)$ & $\begin{array}{l}\Delta / \text { month at } \\
0.5 \mathrm{MeV}(\%)\end{array}$ & $\mathrm{t}_{\frac{1}{2}}$ \\
\hline${ }^{103} \mathrm{Rh}$ & 12694 & 0.17 & 42 seconds \\
${ }^{109} \mathrm{Ag}$ & 11559 & 0.20 & 24 seconds \\
\hline
\end{tabular}

Results of the Geant4 simulation, showing the ratio of electrons produced in the emitter for each material compared to rhodium, are in Table III. Results are listed in order of suitability with tantalum being the top candidate, followed by terbium, thulium, lutetium, rhodium, silver, and finally iridium.

TABLE III

GEANT4 SIMULATION RESULTS SHOWING RATIO OF ELECTRONS PRODUCED IN THE EMITTER TO THAT OF RHODIUM FOR EACH EMITTER CANDIDATE. CANDIDATES ARE LISTED IN ORDER OF JUDGED SUITABILITY.

\begin{tabular}{ll}
\hline Element & Ratio of $\mathrm{e}^{-}$in emitter compared to $\mathrm{Rh}$ \\
\hline $\mathrm{Ta}$ & 2.3 \\
$\mathrm{~Tb}$ & 1.2 \\
$\mathrm{Tm}$ & 1.0 \\
$\mathrm{Lu}$ & 1.5 \\
$\mathrm{Rh}$ & 1.0 \\
$\mathrm{Ag}$ & 0.9 \\
$\mathrm{Ir}$ & 2.7 \\
\hline
\end{tabular}

\section{DISCUSSION}

Overall, tantalum is the most promising emitter candidate for FS-SPND fabrication. It produces a relatively large number of electrons per unit volume and is readily available. It has one naturally occurring isotope, ${ }^{181} \mathrm{Ta}$, and the $\beta$-decay half life of the $(n, \gamma)$ reaction product, ${ }^{182} \mathrm{Ta}$, is 114 days. 114 days is a long enough $\beta$ decay half life as to not add appreciably to the detector response during one fuel cycle of a nuclear reactor. Terbium and thulium will also produce excellent SPND emitters for these same reasons. They are rated lower because they will produce less efficient detectors than tantalum in terms of current produced per unit flux.

While iridium does produce more electrons per unit volume in an SFR flux, it will likely produce a noisier detector response than tantalum. It is for this reason that iridium 
was not rated as the first $\mathrm{c}$ hoice $\mathrm{m}$ aterial. I ridium $\mathrm{h}$ as two stable isotopes, ${ }^{191} \mathrm{Ir}$ and ${ }^{193} \mathrm{Ir}$, with 37.3 and $62.7 \%$ natural abundances, respectively. ${ }^{194} \mathrm{Ir}$, which is the neutron-capture reaction product of the more abundant ${ }^{193} \mathrm{Ir}$, has a $\beta$-decay half life of 19 hours. This is far too short to make iridium an ideal prompt-type emitter candidate and much too long to qualify as an acceptable delayed-type candidate. Additionally, the signal from an iridium detector would likely be significantly more complex than that of tantalum by having a prompt component as well as a 19-hour component and a 7-day component.

At first, lutetium appears to be similar to iridium. It has two stable isotopes: ${ }^{175} \mathrm{Lu}$, which is $97.4 \%$ abundant, and ${ }^{176} \mathrm{Lu}$, which is $2.6 \%$ abundant. Because ${ }^{175} \mathrm{Lu}$ neutron captures to stable ${ }^{176} \mathrm{Lu}$, the signal would for the most part be entirely prompt. This is because it would take a significant number of neutron captures for the bulk of the material to transmute to

${ }^{177} \mathrm{Lu}$, which has a $\beta$-decay half life of 6.6 days. It is for this reason that lutetium would still make a suitable SPND emitter.

The two delayed-type candidates, rhodium and silver, produce a lower number of electrons per unit volume than most of the other prompt-type candidates, a fact that runs contrary to the rule of thumb for existing thermal-spectrum SPNDs. Rhodium is the best candidate of the two because it has only one stable isotope, ${ }^{103} \mathrm{Rh}$, the neutron-capture reaction product of which, ${ }^{104} \mathrm{Rh}$, has a sufficiently short half life of 42 seconds for a delayed-type SPND. The $\beta$-decay daughter of ${ }^{104} \mathrm{Rh}$ has a $\mathrm{Q}_{\beta}$ value of $2.44 \mathrm{MeV}$ and $98 \%$ feeding to the ground state. Any $\beta$ particles produced by this branch of the decay will likely have enough energy to escape to the collector to produce a signal in the detector.

Alternatively, silver will produce less electrons per unit volume, and it also has two stable isotopes: ${ }^{107} \mathrm{Ag}$ (51.8\% abundant) and ${ }^{109} \mathrm{Ag}(48.2 \%$ abundant $)$.

Both of the neutron-capture reaction products have short enough $\beta$-decay half lives for suitable delayed-type SPND at 2.4 minutes and 24.6 seconds, respectively. However, the detector response function from this emitter would be more complicated than rhodium, so it is less ideal as a delayed type emitter.

\section{CONCLUSIONS}

The calculated burnup rates for these chosen emitter candidates in a fast-neutron flux a re c omparable $t$ o $t$ hose of existing high-sensitivity emitters operating in thermal-neutron fluxes [8]. Therefore, it is expected that e ach of the identified candidates will produce a suitably sensitive detector for this application. However, these results are preliminary, as further work remains to be done to simulate these detectors with optimized geometries and to examine the radiation hardness of these materials.

\section{ACKNOWLEDGMENTS}

This work was conducted in conjunction with the VTR project and is based on work supported by the US Department of Energy under Prime Contract No. DE-AC05-00OR2272 to Oak Ridge National Laboratory. Any opinions, findings, and conclusions or recommendations expressed in this publication are those of the authors and do not necessarily reflect the views of the US Department of Energy or Oak Ridge National Laboratory.

\section{REFERENCES}

[1] G. F. KNOLL, Radiation Detection and Measurement, John Wiley \& Sons (2010).

[2] N. GOLDSTEIN and W. TODT, "A Survey of Self-Powered DetectorsPresent and Future," IEEE Transactions on Nuclear Science, 26, 1, 916923 (1979).

[3] M. CHADWICK, M. HERMAN, P. OBLOŽINSKÝ, ET AL., "ENDF/BVII.1 Nuclear Data for Science and Technology: Cross Sections, Covariances, Fission Product Yields and Decay Data," Nuclear Data Sheets, 112, 12, 2887 - 2996 (2011), Special Issue on ENDF/B-VII.1 Library.

[4] BNL, NNDC, https://www.nndc.bnl.gov/nudat2/.

[5] V. VERMA, L. BARBOT, P. FILLIATRE, C. HELLESEN, C. JAMMES, and S. J. SVÄRD, "Self powered neutron detectors as in-core detectors for sodium-cooled fast reactors," Nuclear Instruments and Methods in Physics Research Section A: Accelerators, Spectrometers, Detectors and Associated Equipment, 860, 6-12 (2017).

[6] C. ALLAN, "Experimental measurements of neutron and gamma-ray sensitivities of flux detectors having a mixed response," IEEE Transactions on Nuclear Science, 29, 1, 660-664 (1982).

[7] J. J. DUDERSTADT, Nuclear Reactor Analysis, Wiley (1976).

[8] W. TODT, "Characteristics of self-powered neutron detectors used in power reactors," Core Instrumentation and Core Assessment, Nuclear Energy Agency, Boulogne-Billancourt, France (1996).

[9] S. J. ZINKLE and G. WAS, "Materials challenges in nuclear energy," Acta Materialia, 61, 3, 735-758 (2013).

[10] S. GLADSTONE and A. SESONSKE, "Nuclear Reactor Engineering: Reactor Design Basics," (1994).

[11] E. MENDOZA and D. CANO-OTT, "Update of the Evaluated Neutron Cross Section Libraries for the Geant4 Code (see also INDC (NDS)0612)," Tech. rep., International Atomic Energy Agency (2018).

[12] D. WIARDA, M. DUNN, N. GREENE, M. WILLIAMS, C. CELIK, and L. PETRIE, "AMPX-6: a Modular Code System for Processing ENDF/B," (2016). 